\title{
Interfacial tension in water/n-decane/naphthenic acid systems predicted by a combined COSMO-RS theory and pendant drop experimental study
}

Olesen, Kristian B.; Pedersen, Anne-Sofie Dahl; Nikolajsen, Lasse V.; Andersson, Martin P.; Sølling, Theis I.; Sauer, Stephan P. A.; Mikkelsen, Kurt V.

Published in:

Molecular Physics

Link to article, DOI:

$10.1080 / 00268976.2020 .1764645$

Publication date:

2020

Document Version

Peer reviewed version

Link back to DTU Orbit

Citation $(A P A)$ :

Olesen, K. B., Pedersen, A-S. D., Nikolajsen, L. V., Andersson, M. P., Sølling, T. I., Sauer, S. P. A., \& Mikkelsen, K. V. (2020). Interfacial tension in water/n-decane/naphthenic acid systems predicted by a combined COSMORS theory and pendant drop experimental study. Molecular Physics, 118(18), [e1764645 ].

https://doi.org/10.1080/00268976.2020.1764645

\section{General rights}

Copyright and moral rights for the publications made accessible in the public portal are retained by the authors and/or other copyright owners and it is a condition of accessing publications that users recognise and abide by the legal requirements associated with these rights.

- Users may download and print one copy of any publication from the public portal for the purpose of private study or research.

- You may not further distribute the material or use it for any profit-making activity or commercial gain

- You may freely distribute the URL identifying the publication in the public portal 


\section{Interfacial tension in water/n-decane/naphthenic acid systems predicted by a combined COSMO-RS theory and pendant drop experimental study}

Received 00th January 20xx, Accepted 00th January 20xx DOI: $10.1039 / \times 0 \times x 00000 x$
Kristian B. Olesen ${ }^{\mathrm{a}}$, Anne-Sofie Dahl Pedersen ${ }^{\mathrm{a}}$, Lasse V. Nikolajsen ${ }^{\mathrm{a}}$, Martin P. Andersson ${ }^{\mathrm{b}}$, Theis I. Sølling ${ }^{c}$, Stephan P. A. Sauer ${ }^{\mathrm{a}}$, and Kurt V. Mikkelsen ${ }^{\mathrm{a}}$

\section{Introduction}

Consideration of interfacial phenomena is important to many chemical industries, and crucial to several subdivisions of the oil and gas industry. ${ }^{1}$ Essentially, after conventional recovery methods, the residual oil in the reservoir is either inaccessible due to reservoir heterogeneities or retained in the rock due to capillary forces. $^{2}$ The magnitude of the capillary forces is proportional to the interfacial tension of the oil/water system ${ }^{3}$ and some more effective extraction methods rely on decreasing the interfacial tension ${ }^{4-7}$ making it worthwhile to investigate liquid-liquid interfacial phenomena.

Experimentally, interfacial tension can be measured using several techniques, including, pendant drop measurements. ${ }^{8}$ In contrast, computational modelling of interfacial tension is most often done using a single method - molecular dynamics. This method has been applied to model the surfactant structure at interfaces, ${ }^{9,10}$ the effect of supercritical $\mathrm{CO}_{2}$ on the interfacial tension, ${ }^{11,12}$ and the effect of aqueous ions on interfacial tension. ${ }^{13}$

However, it was recently proposed by Andersson et al., ${ }^{14}$ to utilise the COSMO-RS solvation theory in conjunction with a. Department of Chemistry, University of Copenhagen, Copenhagen, Denmark. b. Department of Chemical and Biochemical Engineering, Technical University of Denmark, Kgs. Lyngby, Denmark.

c. Center for Integrative Petroleum Research, King Fahd University of Petroleum \& Minerals, Dhahran, Saudi Arabia. density functional theory (DFT) quantum mechanical calculations to compute the interfacial tension several orders of magnitude faster than molecular dynamics simulations. COSMO-RS theory, developed by Klamt et al. ${ }^{15-17}$ as an extension of their original COSMO dielectric continuum solvation model, has already shown its great applicability to solvation phenomena. ${ }^{18}$ Some examples thereof are the good predictions of COSMO-RS theory of aqueous $\mathrm{pK}_{\mathrm{a}},{ }^{19}$ nonaqueous $\mathrm{pK}_{\mathrm{a}}{ }^{20}$ and adsorption constants and enthalpies of adsorption for organic compounds at an air-water interface. ${ }^{21}$ Also, the theory has been used for predicting changes in $\mathrm{pK}_{\mathrm{a}}$ of organic acids and bases when at an organic/aqueous interface. ${ }^{22}$

Our research utilises COSMO-RS and pendant-drop measurements to investigate the interfacial tension of water/n-decane/surfactant systems. The surfactants chosen are five members of a homologous series of model naphthenic acids: cyclohexane carboxylic acid, cyclohexyl acetic acid, 3cyclohexyl propionic acid, 4-cyclohexyl butyric acid, and 5cyclohexyl valeric acid. (See Figure 1).

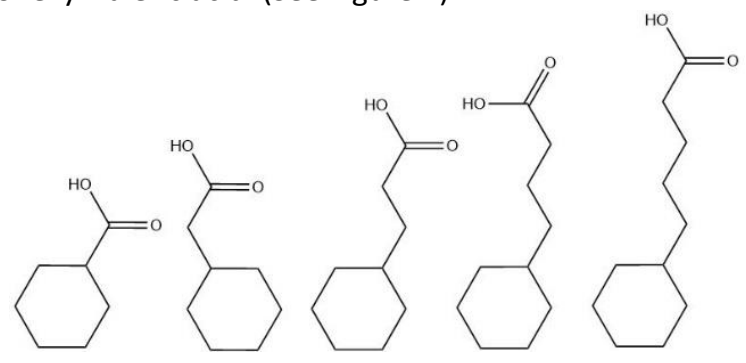

Figure 1: Model naphthenic acids included in the study. From left to right: Cyclohexane carboxylic acid, cyclohexyl acetic acid, 3-cyclohexyl propionic acid, 4-cyclohexyl butyric acid, 5-cyclohexyl valeric acid. These acids are crude oil. $^{2}$ 
Therefore, this study seeks to answer three questions: What are the interfacial tensions of the five model water/n-decane systems containing solvated naphthenic acids as surfactants, and can COSMO-RS accurately predict the interfacial tension of these? Lastly, the aim is to arrive at an understanding of how how the length of the surfactant's hydrophobic tail impacts interfacial tension?

\section{Computational methods}

\section{COSMO-RS solvation theory for prediction of interfacial tension}

The COnductor-like Screening Model for Real Solvents (COSMO-RS) solvation theory is an extension to the COnductor-like Screening Model (COSMO) dielectric continuum model. The COSMO solvation model assumes that the solute is embedded in a conductor representing the solvent. This greatly simplifies the calculation of the screening charge densities acting onto the segmented molecular surface using computational methods. ${ }^{15}$ COSMO-RS uses this idealised solvation state as a reference state. Fluids are then treated as ensembles of pairwise interacting surface segments that have a surface charge density. The composition of an ensemble is determined by the molecular composition of the fluid and the screening charge densities of the surface segments of the molecules in the fluid. ${ }^{16}$ Applying statistical thermodynamics to this ensemble allows for the calculation of chemical potentials of solutes in solvents relative to the ideally screened reference state. ${ }^{17}$ From the chemical potential, the two-phase partitioning of solutes, and solvation energies amongst others can be calculated. COSMO-RS calculates energies relative to the reference state using only inputs from the original quantum mechanical calculation. Thus, COSMO-RS calculations can be performed using maximum two DFT calculations for each species in the system (gas + conductor reference state).

Because COSMO-RS treats a molecule as an ensemble of charged surface segments it enables the calculation of the chemical potential of a solute at a flat interface when it is interacting with two phases. This is achieved by letting one portion of the molecule's surface segments interact with one

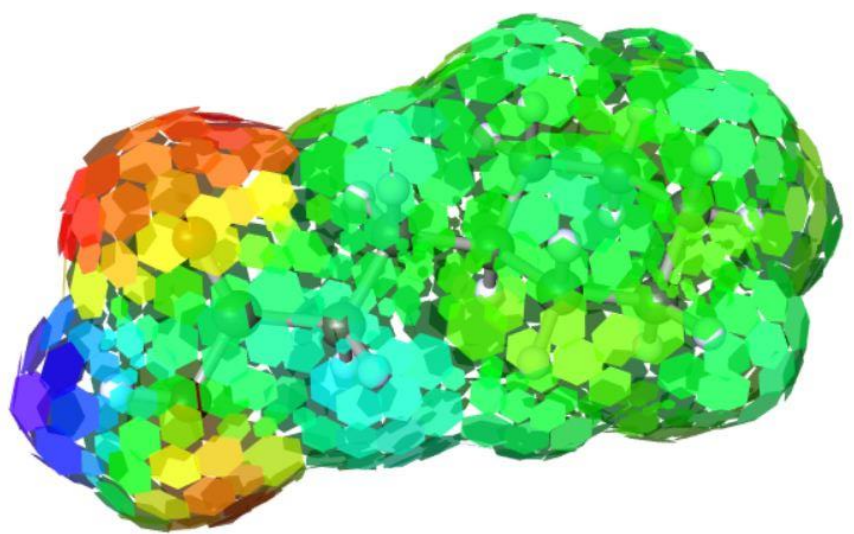

Figure 2: Visualisation of the segmented surface of 3-cyclohexyl propionic acid. The color indicates the surface charge density of the surface segme
red and blue represent negative and positive charges respectively.

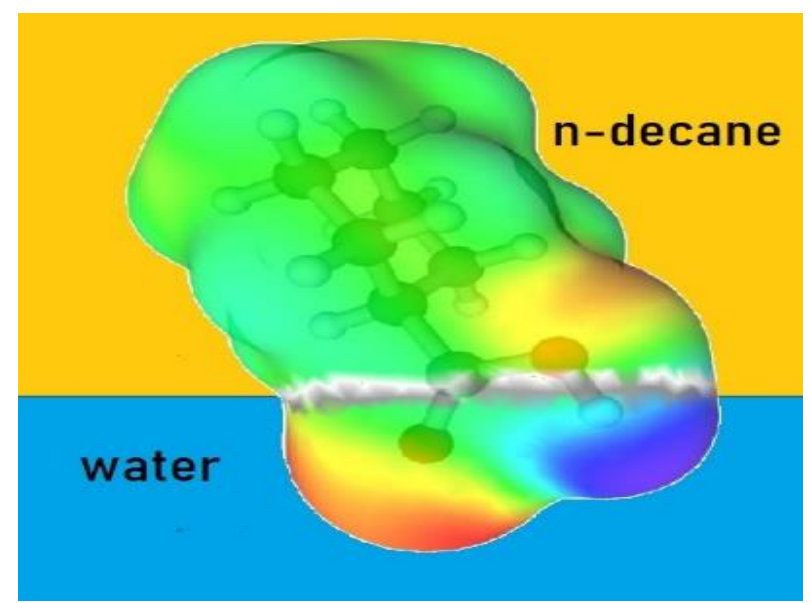

Figure 3: Illustration of the COSMO surface of cyclohexane carboxylic, where one part is interacting with the organic phase, while the other is where one part is interacting with the
interacting with the aqueous phase.

phase, while the other interacts with the other phase (Figure 2). ${ }^{23}$ Andersson et al. ${ }^{14}$ utilised this interfacial solvation energy calculation along with the calculation of the two-phase equilibrium composition of the system to calculate the interfacial tension of systems in an iterative manner. The same method is applied in this study.

\section{Conformer search}

Molecules were drawn using Avogadro ${ }^{24,25}$ software with a subsequent MMFF9426-30 geometry optimization. Then, for each molecule, conformers were generated using the Open Babel Confab code. ${ }^{31}$ The geometries of the generated structures were then optimized using the semi-empirical PM7 method $^{32}$ in Gaussian16. ${ }^{33}$ Optimized structures that did not terminate normally, were discarded. After this they were sorted by Root-Mean-Square-Deviation using the Kabsch algorithm. ${ }^{34,35}$ Structures with RMSD below 0.1 were removed to identify unique structures and to avoid artificial degeneracies in the following Boltzmann distribution calculation. In this calculation, conformers amounting to less than $0.5 \%$ of the population were removed. An overview of the sorting process is outlined in the supporting information. A total of 3, 6, 24, 44, 71 conformations for the five carboxylic acids, as sorted by increasing chain length, was found.

\section{DFT calculations}

The resulting conformations were geometry optimized, as embedded within a conductor, i.e. using the COSMO assumption with infinite dielectric constant. It was confirmed from analysis of vibrational frequencies that all optimised geometries represented energetic minima. The DFT calculations were performed using the TURBOMOLE software package, ${ }^{36,37}$ in which, COSMO has been implemented. ${ }^{38}$ The DFT calculations utilised the BP86 exchange-correlation functional ${ }^{39,40}$ and def-TZVP basis set. ${ }^{41,42}$

\section{COSMO-RS calculations}

COSMO-RS calculations were performed using the COSMOtherm program, ${ }^{43,44}$ For the calculations, water and $n$ - 
decane COSMO-files were found in the COSMOtherm database at the BP-TZVP level, while the carboxylic acids were generated from the DFT calculations. The chosen parameterisation was BP_TZVP_C30_1601.45 Using COSMOtherm, the phase partitioning of the individual carboxylic acids was calculated as expressed by the logP of the distribution of the acids between the $\mathrm{n}$-decane phase and the water phase. Calculation of interfacial tension was done using the method and implementation as developed by Andersson et al. ${ }^{14}$ These calculations, were executed using a computer program written in the python language by Nikolajsen et al. ${ }^{46}$ in accordance with the method of Andersson et al. The program utilises the liquid-liquid extraction (LLE) and flatsurf modules of the COSMOtherm program to calculate the interfacial tension of the system. The calculations were done at a concentration of acid in $\mathrm{n}$-decane of $1.66 \mathrm{~mol} \%$.

In order to keep the mass balance in the system, the liquid liquid extraction calculation was based on mole numbers. Generally, the COSMO-RS calculations of the interfacial tension converged in a matter of minutes for the systems considered.

\section{Experimental methods}

\section{Interfacial tension instrumentation}

The interfacial tensions were measured with the pendant drop technique using the Attension Theta Optical Tensiometer instrument from Biolin Scientific. The instrument records images of a drop suspended from a needle into another fluid with which the drop does not mix. From the shape of the drop, the interfacial tension can be calculated when the density difference between the two phases are known. ${ }^{47}$ The mathematical relation is given in Eq. 1.

$$
\gamma=\frac{\Delta \rho g r_{o}^{2}}{\beta}
$$

Where the interfacial tension is $\gamma$ and $\Delta \rho$ is the density difference between the liquid phases, $g$ is the gravitational constant, $r_{o}$ is the radius of curvature at the drop's apex, while $\beta$ is a shape parameter that is unique for each drop shape and is determined by the computer software. ${ }^{48,49}$

Density measurements of the surfactant solutions and water were taken on an Anton Paar DMA $4500 \mathrm{M}$ density meter, and it was measured at $22^{\circ} \mathrm{C}$ - the temperature of the laboratory. Measured densities are shown in supporting information.

\section{Chemicals}

The five carboxylic acids were acquired from Sigma-Aldrich and used as delivered. Purities of the acids were 98\%, 98\%, 99\%,
$99 \%$, and $98 \%$, respectively, for homologues 1 through 5 . ndecane was from local stock and was purified by passing it through a clean silica column four times. After this procedure, the interfacial tension was measured and found to be stable over 600 seconds, signifying no noteworthy diffusion of surface-active impurities to the surface. For the measurements, Milli- $\mathrm{Q}^{\circledR}$ water (Resistivity of $18.2 \mathrm{M} \Omega \mathrm{cm}$ ) was used.

Surfactant solutions were prepared by weighing out the appropriate amount of the liquid carboxylic acid into a clean container and adding the purified decane using a clean glass pipette until a concentration of 1.66 mol\% was reached.

\section{Procedure}

Measurements were conducted by immersing a $500 \mu \mathrm{L}$ gastight syringe with a J-shaped needle into a cubic quartz cell containing Milli- $\mathrm{Q}^{\circledR}$ water. Drops of surfactant solution or pure $\mathrm{n}$-decane were created manually with the syringe and measurements were recorded using the OneAttension software. Measuring on drops that are close to the maximum volume that can be sustained improves the precision of the method, 50 and drops were measured at volumes around $95 \%$ of the maximum volume. For each system, three drops were measured, and, after forming the drop, it was repeatedly imaged every fourth second yielding a total of 46 images over a period of $180 \mathrm{~s}$. The experimental data for each system is thus three replicate measurements, each consisting of 46 measured IFT values. In between measurements on different salt solutions, the quartz cell and needle were cleaned, before the quartz cell was filled with a new aqueous phase. IFT values for pure water against surfactant solutions were conducted for all five acids. For cyclohexane propanoic acid the IFT value decreased by a unit before stabilizing after ten minutes, after which the drop was imaged repeatedly over $180 \mathrm{~s}$.

\section{Statistical data treatment}

Obtained data sets were checked for outliers using the twotailed Grubb's test for outliers ${ }^{51}$ at a confidence level of $95 \%$. Using the Shapiro-Wilk test for normality ${ }^{52}$ with an alpha level of 0.05 it was found that around $95 \%$ of data sets were normal distributed. Hence, all data sets were assumed to be normal distributed. Uncertainties were expressed as $99.5 \%$ confidence intervals computed from the standard deviation of the measurements. The uncertainty on the system's average IFT was calculated as the square root of the sum of the squared uncertainties of each replicate as per standard error propagation procedures.

\section{Accuracy and precision}


The accuracy of the method was tested by measuring the IFT between pure $n$-decane and water. Obtained average IFT results for four measurements measured over $600 \mathrm{~s}$ were 51.5 $\mathrm{mN} / \mathrm{m}, 52.0 \mathrm{mN} / \mathrm{m}, 51.4 \mathrm{mN} / \mathrm{m}$, and $51.2 \mathrm{mN} / \mathrm{m}$. Compared to literature values in the range of $51.1 \mathrm{mN} / \mathrm{m}$ to $53.2 \mathrm{mN} / \mathrm{m}$, the accuracy is very good. ${ }^{53-57}$ Uncertainties were found to be between $\pm 0.1 \mathrm{mN} / \mathrm{m}$ and $\pm 0.3 \mathrm{mN} / \mathrm{m}$ when calculated as described in Statistical Data Treatment. Additionally, by using repeated measurements on the same system, it was found that measured values could vary up to $\pm 0.23 \mathrm{mN} / \mathrm{m}$ simply from small changes in the position of the needle when repositioning it after the cleaning procedure. Consequently, the total uncertainty of the systems in this study are given simply as the sum of this uncertainty and the random uncertainties mentioned in Statistical Data Treatment. The simple summation of uncertainties allows for a wider margin of error on the average value.

\section{Results}

\section{Phase partitioning}

The calculated partition coefficients (Table 1) reveal that in two phase systems, where the carboxylic acid is initially solubilised in the $n$-decane phase, most of the acids are best solubilised in the organic phase. The only exception is cyclohexane carboxylic acid, where more of the acid is predicted to diffuse into the aqueous phase than is retained in the organic phase. Also, expectedly, the water solubility decreases as the chain length of the hydrophobic tail of the acid surfactants increase.

\section{Calculated IFT}

Table 1 shows the calculated interfacial tension values for the five different systems each containing one carboxylic acid in 1.66 mol\% relative to the $n$-decane phase. The results are from calculations using the absolute number of moles to represent the composition of the system. Looking at the trend in IFT when increasing the chain length of the surfactant, the smallest acid cyclohexane carboxylic acid is predicted to produce the highest interfacial tension, while there seems to be a minimum at 3-cyclohexyl propionic acid, before the interfacial tension increases again for the two largest acids. The minimum at 3-cyclohexyl propionic acid is the result of the following. As the oil solubility of the naphthenic acid increases, there is less energetic gain in migrating to the interface, but since it is an equilibrium, the higher concentration in the oil phase fosters migration to the interface. For the aqueous phase, the energetic gain increases with chain length while the concentration decreases. Evidently, there is an optimum between these two developments in both phases resulting in the highest interfacial coverage and, consequently, lowest IFT for 3-cyclohexyl propionic acid.

\section{Comparison with experiment}

Table 1: Calculated values for $\log P$ for the phase partitioning, interfacial tension, and the surface mole fraction from the interfacial tension calculation. Lastly, the experimental IFT is listed as well.

\begin{tabular}{l|cccc}
\hline & \multicolumn{1}{l}{} & & \\
& $\begin{array}{c}\log \mathrm{P} \\
\left(22^{\circ} \mathrm{C}\right)\end{array}$ & $\begin{array}{c}\text { IFT } \\
(\text { Calc. })\end{array}$ & $\begin{array}{c}\text { Surface Mole } \\
\text { Fraction }\end{array}$ & $\begin{array}{c}\text { IFT } \\
(\text { Exp. })\end{array}$ \\
\hline Cyclohexane carboxylic acid & 0.17 & 28.8 & 0.44 & $30.4 \pm 0.4$ \\
Cyclohexyl acetic acid & -0.27 & 25.7 & 0.48 & $28.8 \pm 0.4$ \\
3-cyclohexyl propionic acid & -0.77 & 25.1 & 0.49 & $27.6 \pm 0.3$ \\
4-cyclohexyl butyric acid & -1.3 & 26.0 & 0.48 & $29.6 \pm 0.4$ \\
5-cyclohexyl valeric acid & -1.9 & 26.9 & 0.46 & $28.9 \pm 0.4$ \\
\hline
\end{tabular}

Comparison with the measurements of the interfacial tension can be seen in figure 4 . The quality of the predictions is good, with a mean-absolute-deviation (MAD) of only $2.6 \mathrm{mN} / \mathrm{m}$. Considering that the original error estimate on the predictive method is $\pm 3 \mathrm{mN} / \mathrm{m}^{14}$ and that experimental uncertainties were around $\pm 0.4 \mathrm{mN} / \mathrm{m}$, the experimental interfacial tension is predicted with reasonable accuracy in all cases. The systems with cyclohexane carboxylic acid and 5-cyclohexyl valeric acid are accurately predicted when considering errors on both the calculation and experiment. The predictions are also able to reproduce the trend in the interfacial tension versus the length of the hydrophobic backbone of the carboxylic acid.

\section{Sensitivity Analysis of the IFT calculations}

To probe the sensitivity of interfacial tension towards the concentration of the acid, a series of calculations were done for acid concentrations in the range 0.001-10 mol\%. The results are shown in Figure 5, where it is seen that the concentration dependence is overall quite similar for all the acids in our study. However, subtle differences occur. The slope of the curves in the active region ( $\sim 1 \mathrm{~mol} \%$ acid), i.e. the change in interfacial tension as a function of acid concentration varies systematically with acid size. The concentration dependence is largest for the smallest acid (cyclohexane carboxylic acid) and smallest for the largest acid (5-cyclohexyl valeric acid). Contrary to this observed trend, the concentration at which the interfacial tension begins to be affected by the presence of the acid ( 0.1 mol\%) has the opposite trend, where the largest acid(s) become active at a lower concentration than the smallest acid.

We do not currently know the physical reason for the variations in concentration dependence, but the range of acids we

have

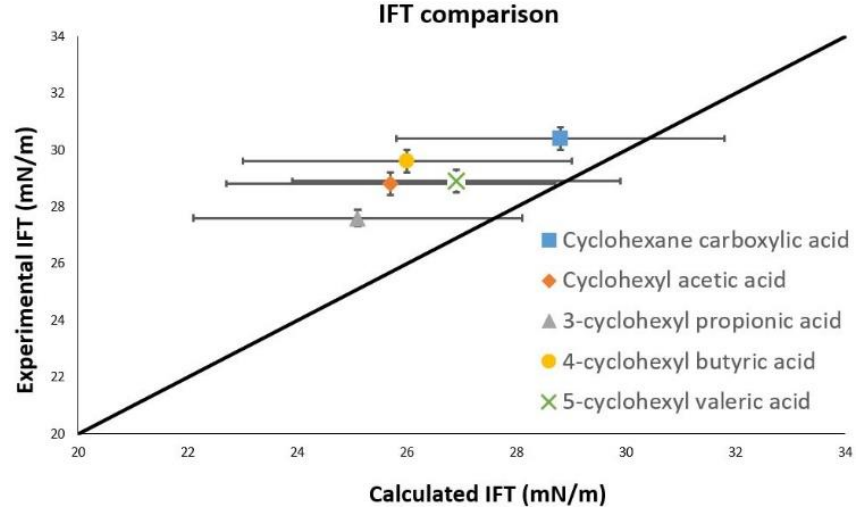

Figure 4: Comparison of calculated and experimental interfacial tension values. Error bars are added to express the uncertainty on the experimental value (vertical error bars) and computational values (horizontal error bars). 




Figure 5: Development of interfacial tension versus concentration for the five acids.

chosen have a partition coefficient $\mathrm{P}$ between oil and water that is close to $1(\log (P)=0)$, which could be a contributing factor. It is beyond the scope of this study, but investigating even longer homologues could help understand the observed trends.

\section{Discussion}

The prediction of the interfacial tensions is satisfactory considering the error estimates on both the computational and experimental results. Sayed et al. ${ }^{57}$ investigated the same homologues as this study with the exception of cyclohexyl acetic acid, at similar concentrations and reported interfacial tension values of $29.1 \mathrm{mN} / \mathrm{m}$ for cyclohexane carboxylic acid, while for the propionic-, butyric-, and valeric acid derivatives they report values of $25.4 \mathrm{mN} / \mathrm{m}, 27.8 \mathrm{mN} / \mathrm{m}$, and $28.2 \mathrm{mN} / \mathrm{m}$ respectively. These values are in good agreement with our findings, especially with the predicted IFTs. One thing that should be noted is the timescale of the experimental measurements. COSMOtherm computes thermodynamic properties at equilibrium conditions, while the experimental measurements were conducted over a period of 180 s for each drop. It has previously been found that diffusion of surfactants to surfaces and interfaces and the establishment of equilibrium conditions is seldom complete after $180 \mathrm{~s} 50,58,59$. We monitored the development of interfacial tension versus time while conducting the measurements, which indicates stable interfacial tension in the measuring interval. However, it is quite possible that over longer time scales, the system might reach an equilibrium state with slightly lower interfacial tension values compared to the ones stated in this work, which would agree better with the theoretical predictions.

Another point in the comparison is that our calculations underestimate all the measured interfacial tensions by at least $2 \mathrm{mN} / \mathrm{m}$, which is still smaller than the typical accuracy from IFT predictions using COSMO-RS, $3 \mathrm{mN} / \mathrm{m} .{ }^{14}$ However, as this study considers only five surfactants, it is difficult to assess whether this is a systematic deviation of the COSMO-RS treatment.

One possible source of error is the somewhat naive neglection of dissociation of the acids in the COSMO-RS calculations, as the input for these were the protonated species. Using COSMO-RS, the $\mathrm{pK}_{\mathrm{a}}$ 's of the acids were calculated to be in the range of $4.7-4.8$, and indeed dissociation is possible. Several researchers have noted that the $\mathrm{pK}_{\mathrm{a}}$ of a species at an interface is drastically different from that of the bulk phase, off-set by at least one unit, meaning that species at the interface is deprotonated less severely than would be expected. ${ }^{22,60-62}$ Nevertheless, accounting for dissociation of the acids in the calculation would likely produce even lower IFT values considering the increased affinity of the deprotonated species for the interface. The effect on interfacial tension from deprotonation would be expected to be larger for the smallest (more water soluble) acids, as deprotonation in the oil phase is likely negligible.

As for the speed of the calculations, it is quite clear when considering the preparation of surfactant solutions, calibration of instrument, repeated measurements and the strict requirements to the cleanliness of the apparatus, that determining the interfacial tension of systems experimentally is a quite lengthy procedure relative to the COSMO-RS computations. The real strength of COSMO-RS is in its definition of a reference state and the fact that it requires only a database of DFT calculations for compounds. These two properties in combination allow for very cheap calculations of multiple different thermodynamic properties of fluid mixtures.

\section{Conclusion}

The interfacial tension of five $\mathrm{n}$-decane/water systems containing $\mathrm{n}$-decane solubilised naphthenic acids has been determined by the pendant drop method. They were found to be in the range of $27.6 \mathrm{mN} / \mathrm{m}$ to $30.4 \mathrm{mN} / \mathrm{m}$ showing a non-monotonous development as the length of the surfactant is increased. It was found that for the series of homologues, the lowest interfacial tension was for 3-cyclohexyl propionic acid. The measured values were accurately predicted by the computational method developed by Andersson et al., using density functional theory calculations and the COSMO-RS implicit solvation model. Thus, it has also been shown, that the computationally cheap COSMO-RS calculations predict the interfacial tension values of oil-water-carboxylic acid systems very satisfactorily. The COSMO-RS theory based model is therefore promising and applicable for the efficient and fast screening of multiple interfacial systems.

\section{Conflicts of interest}

There are no conflicts to declare.

\section{Acknowledgements}

KBO is grateful for the travel grant (2018-26) awarded by the Danish Chemical Society. KVM acknowledges support from the Center of Exploitation of Solar Radiation, Department of Chemistry, University of Copenhagen.

\section{References}


L. He, F. Lin, X. G. Li, H. Sui and Z. H. Xu, Chem. Soc. Rev., 2015, 44, 5446-5494. J. Speight, The Chemistry and Technology of Petroleum, CRC Press, Boca Raton, Fifth., 2014.

D. Tiab and E. C. Donaldson, Petrophysics - Theory and Practice of Measuring Reservoir Rock and Fluid Transport Properties, Gulf Professional Publishing, Fourth., 2016. N. R. Morrow and G. Mason, Curr. Opin. Colloid Interface Sci., 2001, 6, 321-337.

S. Strand, D. C. Standnes and T. Austad, Energy \& Fuels, 2003, 17, 1133-1144.

Y. F. Wu, P. J. Shuler, M. Blanco, Y. C. Tang and W. A. Goddard, SPE J., 2008, 13, 26-34.

P. D. I. Fletcher, L. D. Savory, F. Woods, A. Clarke and A. M. Howe, Langmuir, 2015, 31, 3076-3085.

H.-J. Butt, K. Graf and M. Kappl, Physics and Chemistry of Interfaces, Wiley-VCH, First., 2003.

S. S. Jang, S. T. Lin, P. K. Maiti, M. Blanco, W. A. Goddard, P. Shuler and Y. C. Tang, J. Phys. Chem. B, 2004, 108, 1213012140.

J. S. J. Tan, L. Zhang, F. C. H. Lim and D. W. Cheong, Langmuir, 2017, 33, 4461-4476.

B. Liu, J. Shi, M. Wang, J. Zhang, B. Sun, Y. Shen and X. Sun, J. Supercrit. Fluids, 2016, 111, 171-178.

S. Mohammed and G. A. Mansoori, ENERGY \& FUELS, 2018, 32, 5409-5417.

M. Vazdar, E. Pluharova, P. E. Mason, R. Vacha and P. Jungwirth, J. Phys. Chem. Lett., 2012, 3, 2087-2091.

M. P. Andersson, M. V Bennetzen, A. Klamt and S. L. S. Stipp, J. Chem. Theory Comput., 2014, 10, 3401-3408. A. Klamt and G. Schüürmann, J. Chem. Soc. Trans. 2, 1993, 799-805.

A. Klamt, J. Phys. Chem., 1995, 99, 2224-2235.

A. Klamt, V. Jonas, T. Burger and J. C. W. Lohrenz, J. Phys. Chem. A, 1998, 102, 5074-5085.

A. Klamt and F. Eckert, Fluid Phase Equilib., 2000, 172, 4372.

A. Klamt, F. Eckert, M. Diedenhofen, C. Gmbh, B. Str, V. Le and M. E. Beck, 2003, 9380-9386.

F. Eckert, I. V. O. Leito, I. Kaljurand, A. Kütt, A. Klamt and M. Diedenhofen, J. Comput. Chem., 2009, 30, 799-810. C. Mehler, A. Klamt, W. Peukert, AIChE J., 2002, 48, 10931099.

M. P. Andersson, M. H. M. Olsson and S. L. S. Stipp, LANGMUIR, 2014, 30, 6437-6445.

COSMOlogic $\mathrm{GmbH}$ \& Co KG, COSMOtherm Reference Manual v. C3.0, Release 17.01.

M. D. Hanwell, D. E. Curtis, D. C. Lonie, T. Vandermeersch, E. Zurek and G. R. Hutchison, J. Cheminform., 2012, 17. Avogadro: an open-source molecular builder and visualization tool. Version 1.2.0, http://avogadro.cc. T. A. Halgren, J. Comput. Chem., 1996, 17, 490-519. T. A. Halgren, J. Comput. Chem., 1996, 17, 520-552. T. A. Halgren, J. Comput. Chem., 1996, 17, 553-586. T. A. Halgren and R. B. Nachbar, J. Comput. Chem., 1996, 17, 587-615. T. A. Halgren, J. Comput. Chem., 1996, 17, 616-641.
N. M. O'Boyle, T. Vandermeersch, C. J. Flynn, A. R. Maguire and G. R. Hutchison, J. Cheminformatics, 2011, 3:8. K. Throssel and M. J. Frisch, Evaluation and Improvement of Semi-empirical methods I: PM7R8: A variant of PM7 with numerically stable hydrogen bonding corrections, prep.

Gaussian 16, Revision B.01, M. J. Frisch, G. W. Trucks, H. B. Schlegel, G. E. Scuseria, M. A. Robb, J. R. Cheeseman, G. Scalmani, V. Barone, G. A. Petersson, H. Nakatsuji, X. Li, M. Caricato, A. V Marenich, J. Bloino, B. G. Janesko, R. Gomperts, B. Mennucci, H. P. Hratchian, J. V Ortiz, A. F. Izmaylov, J. L. Sonnenberg, D. Williams-Young, F. Ding, F. Lipparini, F. Egidi, J. Goings, B. Peng, A. Petrone, T. Henderson, D. Ranasinghe, V. G. Zakrzewski, J. Gao, N. Rega, G. Zheng, W. Liang, M. Hada, M. Ehara, K. Toyota, R. Fukuda, J. Hasegawa, M. Ishida, T. Nakajima, Y. Honda, O. Kitao, H. Nakai, T. Vreven, K. Throssell, J. A. Montgomery Jr., J. E. Peralta, F. Ogliaro, M. J. Bearpark, J. J. Heyd, E. N. Brothers, K. N. Kudin, V. N. Staroverov, T. A. Keith, R. Kobayashi, J. Normand, K. Raghavachari, A. P. Rendell, J. C. Burant, S. S. Iyengar, J. Tomasi, M. Cossi, J. M. Millam, M. Klene, C. Adamo, R. Cammi, J. W. Ochterski, R. L. Martin, K. Morokuma, O. Farkas, J. B. Foresman and D. J. Fox, Gaussian Inc., Wallingford CT, 2016.

W. Kabsch, ACTA Crystallogr. Sect. A, 1976, 32, 922-923. W. Kabsch, ACTA Crystallogr. Sect. A, 1978, 34, 827-828. TURBOMOLE V7.0 2015, Development of University of Karlsruhe and Forschungszentrum Karlsruhe $\mathrm{GmbH}$, 19892007, TURBOMOLE Gmbh, since 2007; available from http://www.turbomole.com.

R. Ahlrichs, M. Bar, M. Haser, H. Horn and C. Kolmel, Chem. Phys. Lett., 1989, 162, 165-169.

A. Schafer, A. Klamt, D. Sattel, J. C. W. Lohrenz and F. Eckert, Phys. Chem. Chem. Phys., 2000, 2, 2187-2193. A. D. Becke, Phys. Rev. A, 1988, 38, 3098-3100. J. P. Perdew, Phys. Rev. B, 1986, 33, 8822-8824. A. Schafer, H. Horn and R. Ahlrichs, J. Chem. Phys., 1992, 97, 2571-2577.

F. Weigend and R. Ahlrichs, Phys. Chem. Chem. Phys., 2005, 7, 3297-3305. COSMOlogic GmbH \& Co. KG, COSMOtherm, Version C30, Release 18, http://www.cosmologic.de.

F. Eckert and A. Klamt, AIChE J., 2002, 48, 369-385.

M. P. Andersson, E. Eckert, J. Reinisch and A. Klamt, Fluid Phase Equilib., 2018, 476, 25-29.

Interfacial Tension from Liquid-Liquid Extraction by L. V. Nikolajsen. Python program available from https://github.com/LasseNikolajsen/ift from lle J. M. Andreas, E. A. Hauser and W. B. Tucker, J. Phys. Chem., 1938, 42, 1001-1019. B. Song and J. Springer, J. Colloid Interface Sci., 1996, 184, 64-76.

B. Song and J. Springer, J. Colloid Interface Sci., 1996, 184, 77-91.

J. D. Berry, M. J. Neeson, R. R. Dagastine, D. Y. C. Chan and R. F. Tabor, J. Colloid Interface Sci., 2015, 454, 226-237. F. E. Grubbs and G. Beck, Technometrics, 1972, 14, 847- 
854.

S. S. Shapiro and M. B. Wilk, Biometrika, 1965, 52, 591-611.

53 S. S. Susnar, H. A. Hamza and A. W. Neumann, Colloids Surfaces A Physicochem. Eng. Asp., 1994, 89, 169-180.

A. Goebel and K. Lunkenheimer, Langmuir, 1997, 13, 369372.

A. Georgiadis, J. P. M. Trusler and A. Bismarck, J. Chem. Eng. Data, 2011, 56, 4900-4908.

S. Zeppieri, J. Rodriguez and A. L. Lopez de Ramos, J. Chem. Eng. Data, 2001, 46, 1086-1088. A. M. Sayed, K. B. Olesen, A. S. Alkahala, T. I. Sølling and N. Alyafei, J. Pet. Sci. Eng., 2019, 173, 1047-1052. O. Brandal, J. Sjoblom and G. Oye, J. Dispers. Sci. Technol., 2004, 25, 367-374.

59 A. Bonfillon, F. Sicoli and D. Langevin, J. Colloid Interface Sci., 1994, 168, 497-504.

60 M. P. Andersson and S. L. S. Stipp, J. Phys. Chem. C, 2012, 116, 18779-18787.

61 J. F. Danielli, Proc. R. Soc. Ser. B-Biological Sci., 1937, 122, 155-174.

62 K. Spildo and H. Hoiland, J. Colloid Interface Sci., 1999, 209, 99-108. 